ISSN 1991-8631

Original Paper

http://indexmedicus.afro.who.int

\title{
Diversité des espèces de mouches des fruits (Diptera : Tephritidae) dans un verger mixte dans la localité de Malang (Ngaoundéré, Cameroun)
}

\author{
Léonard NGAMO TINKEU ${ }^{1 *}$, Donatien LADANG ${ }^{1}$, Jean-François VAYSSIERES ${ }^{2}$ et \\ Jean Paul LYANNAZ ${ }^{3}$ \\ ${ }^{1}$ Université de Ngaoundéré, Faculté des Sciences, BP 454 Ngaoundéré - Cameroun. \\ ${ }^{2}$ CIRAD, UPR HortSys, Montpellier, France-34398; IITA, Cotonou, Bénin. \\ ${ }^{3}$ IRAD-CIRAD, Station polyvalente de Garoua, BP 415 Garoua - Cameroun. \\ *Corresponding author, E-mail: leonard.ngamo@gmail.com
}

\section{RESUME}

La présente étude avait pour but d'inventorier les espèces de mouches des fruits (Diptera : Tephritidae) inféodées au manguier dans la localité de Malang, située dans la région de l'Adamaoua (Ngaoundéré, Cameroun), en zone Soudano-Guinéenne. Pour cela, nous avons identifié les principales espèces présentes dans le verger grâce à un piégeage de détection d'avril 2008 à avril 2009, et, d'autre part, nous avons cherché à connaître les espèces responsables des dégâts à partir du ramassage des mangues dans le verger. Au total, 83052 adultes de mouches des fruits appartenant à neuf espèces ont été capturés et huit espèces ont été déterminées. L'espèce invasive Bactrocera invadens Drew Tsuruta \& White est la plus abondante dans les pièges (66,55\% des captures), suivie de Dacus punctatifrons (Karsch) (29,92\% des captures). La troisième espèce par ordre d'importance est Ceratitis cosyra (Walker) (3,38\% des captures). Six autres espèces sont présentes mais en très faible effectif (avec au total $0,15 \%$ des captures). Les adultes de Tephritidae qui ont émergé des mangues mûres ramassées sous les arbres appartiennent à deux espèces B. invadens $(98,5 \%)$ et $D$. punctatifrons $(1,5 \%)$. Le pic de pullulation de $B$. invadens coïncide avec celui des mangues arrivées à maturité alors que $D$. punctatifrons est surtout présent après la période de maturité des mangues. $B$. invadens est la principale espèce de Tephritidae responsable des dégâts sur mangues à Malang. Les organisations de protection phytosanitaire doivent mettre en oeuvre dans les meilleurs délais des programmes de lutte intégrée afin de pouvoir réduire les dégâts de cette nouvelle espèce invasive en dessous d'un seuil économique de nuisibilité.

(c) 2010 International Formulae Group. All rights reserved.

Mots clés: Mouches des fruits, Mangifera indica, Bactrocera invadens, Dacus punctatifrons, Cameroun, Adamaoua, dynamique des populations.

\section{INTRODUCTION}

Ces dernières années, le secteur horticole est devenu un des éléments importants de la politique de développement agricole dans de nombreux pays d'Afrique Sub-Saharienne. Les exportations horticoles sont considérées comme étant un moteur essentiel de la croissance économique des pays en voie de développement. A cause de l'engagement important des petits producteurs dans la filière horticole, le renforcement de ce secteur est susceptible 
de générer une augmentation substantielle des revenus du monde paysan ainsi que la pérennité de l'emploi au niveau du monde rural. En outre, il contribue à assurer la sécurité alimentaire et permet d'atteindre un meilleur équilibre nutritionnel.

Parmi les fruits tropicaux, la mangue est un de ceux qui ont le plus grand potentiel. L'augmentation de la demande de ce fruit au niveau des marchés mondiaux a produit une augmentation de la production dans des pays africains qui n'avaient pas au départ vocation à être des pays producteurs. Ces pays ont diversifié leur production de cultures non traditionnelles essentiellement pour satisfaire la demande croissante de fruits tropicaux en Europe. La production de mangues en Afrique de l'Ouest, avec plus d'un million de tonnes par an en 2008 (FAO, 2010) est supérieure à celle des agrumes. Cependant, la valorisation de ce potentiel reste actuellement limitée. Plus particulièrement, la capacité des exportateurs Ouest Africains dépend de leur capacité à assurer un flux régulier de fruits (aspect quantitatif) mais aussi respectant les normes standard de qualité des pays importateurs (aspect qualitatif).

Les études conduites sur le manguier en Afrique ces dernières années ont permis de mettre en évidence de nombreux problèmes d'ordre phytosanitaire: d'ordre physiologique, d'ordre phytopathologique et d'ordre entomologique (mouches des fruits, cochenilles, termites, charançon du noyau). Dans la zone de production de l'Adamaoua au Cameroun, la contrainte majeure est liée essentiellement à l'existence des mouches des fruits (Diptera Tephritidae). Les mouches des fruits sont un complexe de ravageurs qui causent les dégâts les plus importants aux mangues dans les zones tropicales (White \& Elson-Harris, 1992). L'arrivée d'une nouvelle espèce invasive, Bactrocera invadens, signalée en 2004 au Cameroun (Ndzana et al., 2008), a considérablement aggravé la pression phytosanitaire exercée par les mouches des fruits sur la production camerounaise de mangues.
Les mouches des fruits ou Tephritidae sont un des groupes de ravageurs qui occasionnent les dégâts les plus importants sur les cultures fruitières, Les mouches de fruits piquent les mangues à l'aide de leur ovipositeur et déposent à faible profondeur à la fois leurs œufs et des bactéries induisant la dégradation rapide de la pulpe (White et Elson-Harris., 1992 ; Ekesi et al., 2006).

Les mouches de fruits sont des ravageurs redoutables des mangues et causent des pertes importantes de production (N'Guetta, 1994 ; Vayssières et al., 2000). L'espèce $B$. invadens vient d'être signalée en Afrique (Drew et al., 2005 ; Vayssières et al., 2005), où elle cause des ravages sur les productions de mangues, que ce soit dans les vergers industriels ou dans les manguiers de case. En Afrique, B. invadens a été signalé pour la première fois en 2003 au Kenya (Lux et al., 2003), en Tanzanie (Mwatawala et al., 2004), puis en 2004 au Sénégal (Vayssières, 2004), au Bénin (Vayssières et al., 2005) et au Cameroun (Ndzana et al., 2008), mais également en Côte d'Ivoire (N'Dépo et al., 2009), et au Burkina Faso (Ouedraogo et al., 2010).

Les espèces les plus nuisibles sont Ceratitis cosyra (Walker), C. quinaria (Bezzi) et $C$. silvestrii (Bezzi) qui sont en général les plus précoces et par conséquent les plus préjudiciables (Ekesi et al., 2006 ; Vayssières et al., 2009a). Cette étude est centrée sur la diversité des espèces de mouches des fruits inféodées au manguier en zone Soudano Guinéenne du Cameroun. Elle se focalise sur l'inventaire des espèces de Tephritidae présentes dans le verger de manguiers d'une part par piégeage et d'autre part sur les observations au laboratoire des émergences des mouches des fruits à partir des mangues ramassées dans le verger.

\section{MATERIEL ET METHODES}

Le suivi de la dynamique des populations de mouches des fruits a été 
réalisé à Ngaoundéré d'avril 2008 à avril 2009 dans un verger mixte de manguiers. Pendant cette période, les données climatiques étaient enregistrées. Des attractifs alimentaires et sexuels ont été utilisés et la fréquence de visites des pièges était hebdomadaire. Le renouvellement des attractifs sexuels était mensuel et celui des attractifs alimentaires était hebdomadaire.

\section{Présentation du verger}

Le site de piégeage se trouve dans la partie septentrionale du Cameroun, dans la localité de Malang, près de Ngaoundéré, dans la commune de Ngaoundéré III (Figure 1). Les cordonnées GPS de ce verger mixte sont : $07^{\circ} 27310 \mathrm{~N}, 13^{\circ} 03180 \mathrm{E}$. L'altitude est de $1130 \mathrm{~m}$.

Le verger multivariétal de Malang a une superficie de $81000 \mathrm{~m}^{2}$. C'est en outre un verger mixte, comportant sept espèces fruitières comprenant des manguiers (171 arbres soit 42,64\%), des mandariniers (100 arbres soit 24,94\%), des avocatiers (57 arbres, soit 14,22\%), des citronniers (49 arbres soit $12,22 \%)$, des orangers ( 2 arbres soit $0,5 \%)$, des pamplemoussiers (19 arbres soit 4,74\%) et des goyaviers (3 arbres soit $0,74 \%$ ). Ces fruitiers sont repartis sur une trentaine de lignes régulières, et chacune d'elle compte 18 plantes au maximum, lignes qui sont distantes les unes des autres de 15 mètres. Les variétés de manguiers présentes sur le site sont : Kent (22\%); Julie (16\%); Smith (14\%); Ica I (13\%); Irwin (6\%); Davis Haden (5\%); Springfels (5\%); Brooks (4\%); Ruby (3\%); Romanea (2\%); Eldon (2\%); Zill (1\%); Tommy Atkins $(1 \%)$; Amélie $(0,5 \%)$; Sabot $(0,5 \%)$; les mangues locales $(5 \%)$.

Présentation des pièges et des attractifs
utilisés
$\quad$ Pour suivre les fluctuations de populations des mouches des fruits et dans le cadre d'un piégeage de détection, nous avons utilisé des attractifs sexuels et alimentaires. Les attractifs sexuels sont des paraphéromones servant à attirer les mâles. Les pièges utilisés sont de type Tephritrap
(TPT). Ils contiennent un type d'attractif auquel est associé un insecticide, le DDVP qui fonctionne dans la stratégie 'attract and kill'. Les attractifs sexuels sont des paraphéromones permettant de capturer les mouches des principaux genres d'importance économique: Ceratitis, Bactrocera et Dacus. Les attractifs utilisés sont: le terpinyl acétate (TER) et le trimedlure (TRI) ciblant les cératites; le cuelure (CUE) ciblant les Dacus et Bactrocera; le méthyl-eugénol (MET) ciblant les Bactrocera principalement. Un attractif alimentaire, le torula (TOR), qui a été également utilisé, se compose de protéines hydrosolubles nécessaires au métabolisme des Tephritidae.

\section{Méthode de capture et disposition des pièges}

En début de campagne en avril 2008, 21 pièges étaient répartis dans le verger expérimental de Malang. Chaque piège placé sur un manguier était suspendu au tiers inférieur de la frondaison, à une distance moyenne de $2 \mathrm{~m}$ du centre de l'arbre. Le piège suspendu est relié à une branche de l'arbre perpendiculaire au tronc, par un fil métallique enduit de graisse solide au niveau du tiers supérieur du fil métallique afin de limiter l'activité prédatrice des insectes sur le contenu du piège. Les pièges suspendus étaient placés dans une aire dégagée d'accès facile et à l'abri des rayons directs du soleil.

Les relevés de pièges se faisaient à intervalles réguliers de sept jours. Lors de ces relevés, les pastilles à torula étaient renouvelées, tandis que les paraphéromones des pièges à attractifs sexuels et les insecticides (DDVP) étaient remplacés tous les 28 jours. Les insectes piégés étaient collectés et conservés par date et par piège dans de l'éthanol à $70^{\circ}$, puis ramenés en laboratoire pour identification et comptage.

Une précaution importante à prendre était de ne pas toucher des mains nues les attractifs et à leur renouvellement, 
pour éviter toute contamination. Les restes d'attractifs retrouvés dans les pièges lors du renouvellement hebdomadaire étaient enfouis sous terre.

\section{Enregistrement des paramètres climatiques} L'enregistrement des paramètres climatiques au niveau du verger de Malang était réalisé d'une part par un thermohygromètre de marque $\mathrm{HOBO}$ onset série $\mathrm{H} 8$ qui enregistrait les données de température et d'humidité relative. Ce thermo-hygromètre était placé dans un arbre situé au centre du verger. Il a été programmé de façon à sauvegarder les données à intervalles réguliers de 2 heures, et ceci pendant toute la durée de capture. D'autre part, l'intensité des pluies était suivie quotidiennement à l'aide d'un pluviomètre à lecture directe. Il était placé au voisinage du verger sur un espace bien dégagé de la végétation alentour.

\section{Suivi de l'émergence des mouches des fruits parasités}

Pendant la période de production des mangues, 25 fruits mûrs pourrissants sans trous de sortie étaient ramassés, une fois par quinzaine sous les manguiers et rapportés au laboratoire où ils étaient pesés et observés séparément dans des seaux de deux litres de capacité, rempli au tiers par du sable fin humide. Les larves de mouches parasitant ces fruits mûrs pourrissants se sont transformées en pupes dans le sable humide et les adultes ont émergé une dizaine de jours après. Les seaux étaient recouverts par une toile moustiquaire et tenu par un fil élastique. Tous les adultes ayant émergés étaient conservés.

\section{Identification des mouches de fruits collectés}

Les mouches collectées dans les pièges ou celles émergeant des pupes issues des élevages en laboratoire ont été regroupées en morphotypes. Ce premier tri a été fait sur la base de la clé pratique de détermination des mouches des fruits d'importance économique. Les identifications des espèces courantes ont été faites au Biological Control Center for
Africa (IITA-Cotonou, Bénin) et au service d'identification des insectes de l'IITA, (station du Cameroun). La confirmation de ces identifications et la détermination des espèces jusque là indéterminées a été faite à la Section Entomologie du Musée Royal d'Afrique Centrale en septembre 2009.

\section{RESULTATS ET DISCUSSION \\ Diversité des espèces de mouches de fruits capturées}

Sur une période de capture de 12 mois, 83.052 mouches de fruits ont été capturées dans le verger mixte de Malang (Tableau 1). Parmi les neuf espèces de mouches présentes, huit ont été identifiées. Deux espèces ont dominé : Bactrocera invadens Drew Tsuruta \& White représentant $66,55 \%$ des captures et Dacus punctatifrons Karsch représentant 29,92\% des captures. Ceratitis (Ceratalaspis) cosyra (Walker) représentant 3,38\% est la troisième espèce d'importance économique. Les six autres espèces, incluant Celidodacus sp, ont de très faibles effectifs. Elles représentent toutes 0,15\% des captures. Il s'agit de Ceratitis (Pterandrus) anonae Graham; Ceratitis (Pterandrus) rosa Karsch; Ceratitis (Pardalaspis) bremii Guérin-Méneville; Notoma biocolatum Bezzi; Celidodacus sp. et Bactrocera (Zeugodacus) mesomelas (Bezzi).

\section{Les espèces de Tephritidae d'importance économique dans le verger de Malang Bactrocera invadens \\ B. invadens, capturé avec le méthyl-} eugénol, est l'espèce majoritaire qui constitue l'essentiel des captures dans les pièges et des émergences à partir des fruits infestés. Arrivé en Afrique en 2003 (Lux et al, 2003), B. invadens est signalé au Cameroun depuis 2004 (Ndzana et al, 2008). En 2010, il est présent dans la plupart des pays africains tropicaux de la côte atlantique à la côte pacifique. 
Présentée comme une espèce de moyenne altitude (Mwatawala et al., 2006a, 2006b), il domine à Ngaoundéré autour de 1000 mètres d'altitude. Certaines de ses plantes hôtes comme le goyavier (Ndzana et al., 2008), l'avocatier (De Meyer et al., 2002), sont présentes à Ngaoundéré. Il pourrait aussi y avoir d'autres plantes hôtes cultivées et sauvages favorables au développement de cette espèce redoutable. Ainsi, dans la perspective de la mise en œuvre de méthodes de lutte intégrée, il serait judicieux de faire l'inventaire de tous ses hôtes.

\section{Dacus punctatifrons}

Cette espèce est capturée essentiellement par le cuelure et totalement absente dans les pièges avec les autres attractifs sexuels. Cette espèce a déjà été signalée comme ravageur des agrumes en Côte d'Ivoire (N'Dépo et al., 2009). C'est une espèce qui semble polyphage, qui s'attaque aussi bien à des cultures fruitières qu'à des cultures maraîchères. En effet, cette espèce a déjà été signalée comme ravageur de la tomate au Cameroun (Tindo \& Tamò, 1999).

\section{Ceratitis cosyra}

Le terpinyl acétate a permis la capture de $C$. cosyra qui est un ravageur important de la mangue mais qui est représenté ici par des effectifs peu importants. C'est un ravageur majeur de la mangue dans tous les pays d'Afrique sub-saharienne et plus particulièrement dans les zones sèches. En outre, c'est une espèce précoce souvent capturée en début de campagne (Vayssières et al., 2004, 2005).

\section{Fluctuations des populations de mouches de fruits}

Vayssières et al. (2009a) ont montré au Bénin que la conjonction de facteurs abiotiques favorables tels que l'augmentation significative de l'humidité relative, l'arrivée des pluies utiles et de facteurs biotiques tels que l'arrivée à maturité des premiers cultivars précoces du manguier induisait une explosion très rapide des populations de $B$. invadens dans les zones Soudaniennes (sensu lato). La climatologie de ces zones Soudaniennes
Béninoises semble assez proche de la climatologie rencontrée dans l'Adamaoua. La courbe annuelle de la pluviométrie de ces zones Soudaniennes Béninoises est de type unimodal avec une moyenne de $1200 \mathrm{~mm}$ par an.

L'espèce prépondérante pendant la période de mûrissement des mangues est B. invadens. Le pic de pullulation de cette espèce invasive a eu lieu en juillet avec 2079 mouches par piège par semaine (Figure 2). La deuxième espèce présentant un important effectif dans le verger est $D$. punctatifrons avec un pic de pullulation de 1855 mouches par piège par semaine en juillet. C'est une espèce tardive qui domine le verger alors que la campagne mangue est terminée. A cette période, les goyaviers sont en phase de mûrissement et peuvent justifier la présence de ces mouches dans le verger, présence qui se poursuit jusqu'au milieu de la saison sèche en décembre. La troisième espèce, $C$. cosyra, présente en très faible effectif, a eu son pic de pullulation avec 367 mouches par piège par semaine en juin.

La période de productivité des mangues, la pluviosité, les températures et l'humidité relative influent plus ou moins directement l'apparition de ces trois espèces de ravageurs qui prédominent dans le verger mixte de Malang. C. cosyra, apparaît en début de saison de production de mangues, avec des températures minimales de $15{ }^{\circ} \mathrm{C}$ et une humidité relative très faible à faible. La chute des effectifs de cette espèce en pleine période de productivité est suivie d'une explosion des populations de B. invadens. Cette espèce dominante, atteint un pic de pullulation avec la recrudescence des pluies. Vayssières et al. (2009b) ont souligné que $C$. cosyra est une espèce plus fréquente en saison sèche tandis que $B$. invadens est une espèce plus fréquente en saison des pluies. D'après Duyck (2005), les Tephritidae en général sont très sensibles aux variations des conditions environnementales, en particulier de l'humidité relative. 
Inventaire des Tephritidae issues des mangues au laboratoire

$\mathrm{Au}$ cours de sept échantillonnages effectués pendant la période de mûrissement des mangues, du premier mai au premier août 2008 dans le verger de Malang, un total de 140 mangues ont été ramassées et suivies séparément au laboratoire (Tableau 2). Le parasitisme a atteint sa valeur la plus forte en début août avec 23 mouches qui ont émergé des 20 mangues mises en observation. Le
Tableau 2 nous indique que $B$. invadens est responsable de $98,5 \%$ des dégâts sur mangues tandis que $D$. punctatifrons représente seulement $1,5 \%$ des dégâts (Tableau 2).

Par ailleurs, nous avons constaté qu'il n'y a pas de corrélation entre le nombre de mouches qui émergent d'une mangue mûre parasitée et le poids de la mangue (Figure 3).

Tableau 1 : Importance numérique des espèces de mouches des fruits capturées.

\begin{tabular}{llll}
\hline & & Total & \% \\
\hline 1 & Bactrocera invadens & 55271 & 66,5499 \\
2 & Bactrocera mesomelas & 5 & 0,0060 \\
3 & Celodidacus sp & 2 & 0,0024 \\
4 & Ceratitis anonae & 57 & 0,0686 \\
5 & Ceratitis bremii & 16 & 0,0193 \\
6 & Ceratitis cosyra & 2809 & 3,3822 \\
7 & Ceratitis rosa & 36 & 0,0433 \\
8 & Dacus punctatifrons & 24851 & 29,9222 \\
9 & Notoma biocolatum & 5 & 0,0060 \\
\hline \multicolumn{7}{r}{} \\
\hline
\end{tabular}

Tableau 2 : Abondance et diversité des mouches émergeant des mangues mûres collectées dans le verger de Malang pendant leur période de mûrissement de mai à août 2008.

\begin{tabular}{lllllllll}
\hline & \multicolumn{7}{c}{ Date de collecte des mangues mûres en 2009 } \\
\hline & $01 / 5$ & $15 / 5$ & $01 / 6$ & $15 / 6$ & $01 / 7$ & $15 / 7$ & $01 / 8$ & Total \\
Bactrocera invadens & 0 & 0 & 3 & 9 & 9 & 22 & 22 & 65 \\
Dacus punctatifrons & 0 & 0 & 0 & 0 & 0 & 0 & 1 & 1 \\
& 0 & 0 & 3 & 9 & 9 & 22 & 23 & $\mathbf{6 6}$ \\
\hline
\end{tabular}

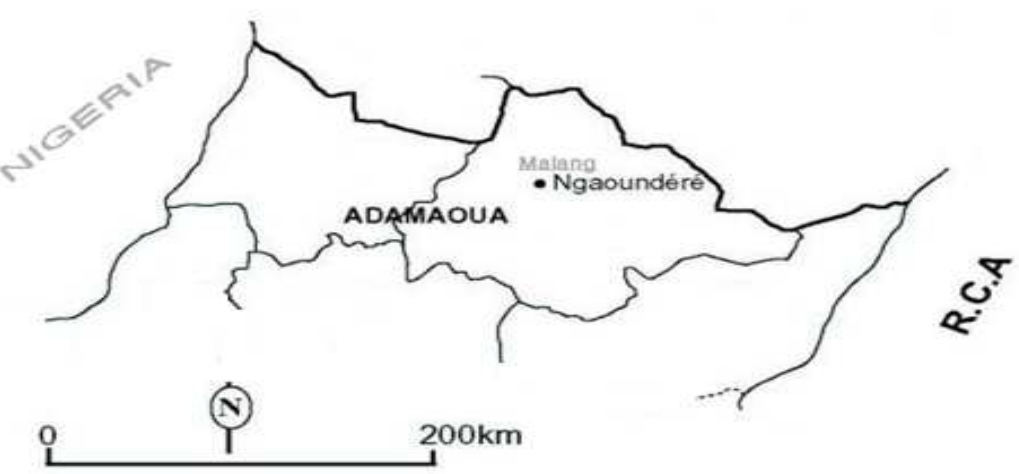

Figure 1: Localisation du site d'étude : Localité de Malang $\left(07^{\circ} 27310 \mathrm{~N}, 13^{\circ} 03180 \mathrm{E}\right)$, Arrondissement de Ngaoundéré, Région de l'Adamaoua. 


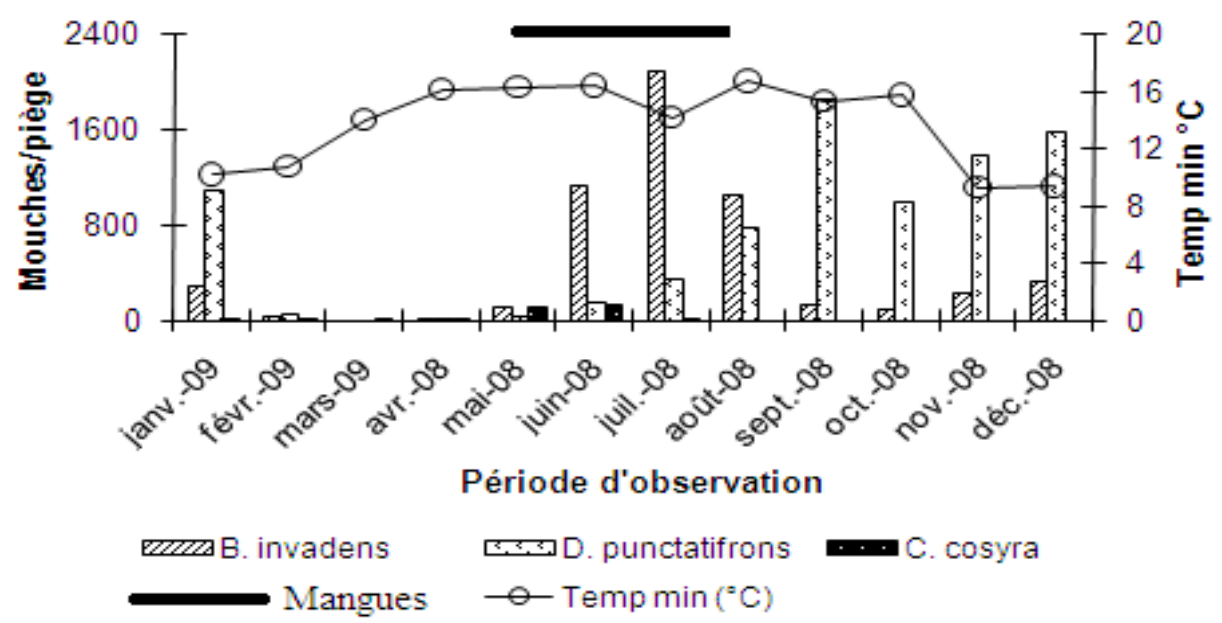

Figure 2: Fluctuation des populations des trois plus importantes espèces de Tephritidae capturées à Malang par piégeage entre avril 2008 et avril 2009.

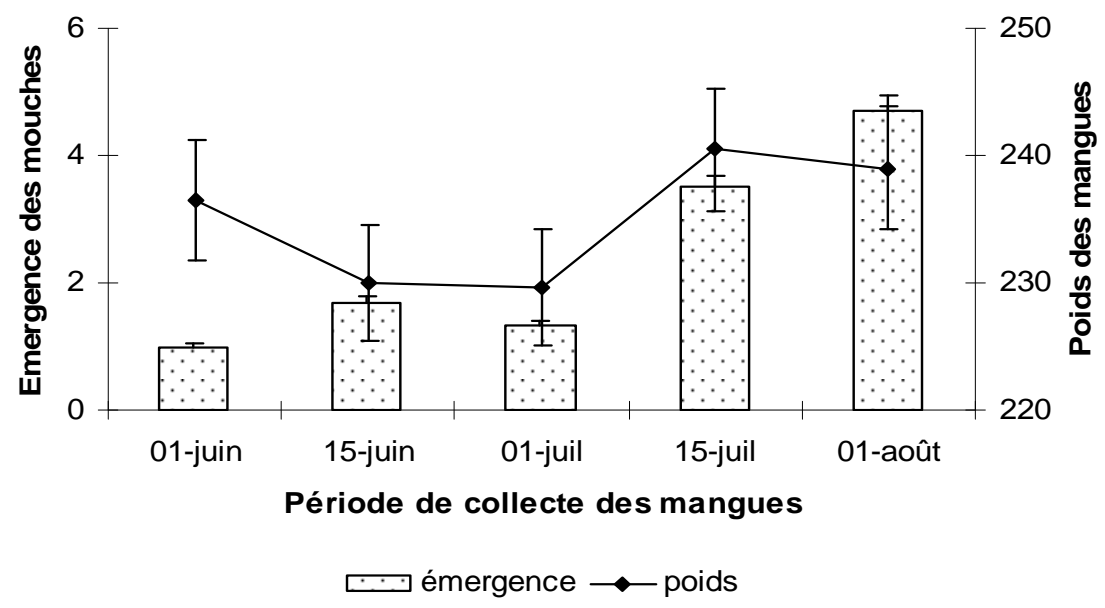

Figure 3: Relation entre le nombre d'adultes qui émergent des mangues infestées et le poids de ces mangues.

\section{Importance de Bactrocera invadens en} Afrique sub-saharienne

Une comparaison de cette étude avec celles déjà effectuées dans le même but au Bénin (Vayssières et al., 2005), au Cameroun (Ndzana et al., 2008) et en Côte d'Ivoire (N'Depo et al., 2009), fait ressortir la présence de plusieurs espèces de mouches de fruits d'importance économique comme ravageurs de la mangue. En général, les espèces du genre Ceratitis sont présentes avant et au début de la montée en puissance des populations de $B$. invadens tant dans les pièges qu'au niveau des infestations des fruits échantillonnés (Vayssières et al., 2005). C. cosyra est un ravageur de la mangue très important dans tous les vergers de manguiers de l'Afrique sub- 
saharienne (Vayssières et al., 2000, 2004, 2005, 2008; Rwomushana et al., 2008; Mwatawala et al., 2009). Il est curieux que nous n'ayons pas eu davantage de dégâts dus à cette espèce avec les fruits ramassés au sol. Un effectif plus conséquent avec des prélèvements hebdomadaires, un échantillonnage de fruits piqués réalisé au sol mais aussi sur les arbres, une période d'échantillonnage de fruits de 3 mois au lieu de 2 nous auraient donné sans doute des résultats plus complets à ce niveau. Une amélioration de la méthode utilisée en récupérant les pupes par tamisage régulier du sable serait également profitable.

B. invadens est donc au Cameroun un ravageur de grande importance économique des mangues et des goyaves (Ndzana et al., 2008). C'est une espèce polyphage redoutable qui s'attaque aussi bien aux goyaves qu'aux mangues et sans doute à de nombreux autres fruits qu'il nous faudra recenser. En effet, il doit y avoir au Cameroun de nombreuses autres plantes hôtes cultivées et sauvages favorables au développement de $B$. invadens. En conséquence, dans la perspective de la mise en œuvre de méthodes de lutte intégrée, il serait judicieux de faire l'inventaire autour du verger, dans un large rayon, de tous ses hôtes qu'ils soient cultivés ou sauvages.

Cette étude préliminaire serait à poursuivre au niveau des récoltes de fruits dans toute la zone de l'Adamaoua afin de mieux connaître l'écologie des principales espèces de mouches des fruits récoltées dans les pièges.

\section{Conclusion}

Trois espèces de Tephritidae ont été mises en évidence dans le verger mixte de Malang, avec par ordre décroissant, $B$. invadens, D. punctatifrons et $C$. cosyra. B. invadens, qui est une espèce nouvelle (décrite en 2005), doit être soigneusement étudiée sur le plan de sa biologie et de son écologie afin de pouvoir mettre au point des méthodes de lutte intégrée (IPM) qui soient efficaces, efficientes et respectueuses des consommateurs et donc de l'environnement. Le Cameroun pourrait s'inspirer des premières méthodes de lutte intégrée qui ont déjà fait leurs preuves au Bénin (Van Mele et al., 2007 ; Vayssières et al., 2009b) dans le cadre du projet WAFFI contre $B$. invadens.

\section{REMERCIEMENTS}

Les travaux ont été menés sur le terrain avec la contribution de M. Alahdji BOUCAR (arboriculteur) et de M. Saïdou MOUSSA (gardien du verger). Qu'ils en soient remerciés. Sur le plan scientifique, nous remercions également le Dr TINDO Maurice du service d'identification de l'IITA et le Dr Marc DE MEYER du Musée Royal d'Afrique Centrale (Tervuren, Belgique). Les auteurs expriment également leur gratitude aux évaluateurs pour leurs commentaires et suggestions pour l'amélioration de la version finale de l'article.

\section{REFERENCES}

De Meyer M, Copeland RS, Lux SA, Mansell M, Quilici S, Wharton R, White IM, Zenz NJ. 2002. Annotated check list of host plants for afrotropical fruit flies (Diptera: Tephritidae) of the genus Ceratitis. Documentation Zoologique, Musée Royal de l'Afrique Centrale, 27: 1 91.

Duyck PF. 2005. Compétition interspécifique et capacités invasives. Le cas des Tephritidae de l'île de La Réunion. Thèse de Doctorat Ph.D, Université Réunion France. 103p.

Drew RAI, Tsuruta T, White IM. 2005. A new species of pest fruit fly (Diptera: Tephritidae: Dacinae) from Sri Lanka 
and Africa. Afr. Entomol., 13: 149-154.

Ekesi S, Nderitu PW, Rwoshmushana I. 2006.

Field infestation, life story and demographic parameters of the fruit flies Bactrocera invadens (Diptera: Tephritidae) in Africa. Bulletin of Entomological Research, 96: 379-386.

FAOSTAT,2010. ttp://faostat.fao.org/site/567

Lux SA, Copeland RS, White IM, Manrakhan A, Billah MK. 2003. A New invasive fruit fly species from the Bactrocera dorsalis (Hendel) group detected in East Africa. Insect Science and Its Application, 23: 355-361.

Mwatawala MW, White IM, Maerere AP, Senkendo FJ, De Meyer M. 2004. A new invasive Bactrocera species (Diptera Tephritidae) in Tanzania. African Entomology, 12: 154-156.

Mwatawala MW, De Meyer M, Makundi RH, Maerere AP. 2006a. Biodiversity of fruit flies (Diptera, Tephritidae) in orchards in different agro-ecological zones of the Morogoro region, Tanzania. Fruits, 61: 321-332.

Mwatawala MW, De Meyer M, Makundi RH, Maerere AP. 2006b. Seasonality and host utilization of the invasive fruit fly, Bactrocera invadens (Diptera: Tephritidae) in central Tanzania, J. Appl. Entomol., 130: 530-537.

Mwatawala MW, De Meyer M, Makundi RH, Maerere AP. 2009. Host range and distribution of fruit-infesting pestiferous fruit flies (Diptera, Tephritidae) in selected areas of Central TanzaniA. Bulletin of Entomological Research, doi:10.1017/S0007485309006695.

N'dépo OR, Hala N, Allou K, Aboua LR, Kouassi KP, Vayssières J-F, De Meyer M. 2009. Abondance des mouches des fruits dans les zones de production fruitières de Côte d'Ivoire: dynamique des populations de Bactrocera invadens. Fruits, 64 : 313-324.
Ndzana Abanda F-X, Quilici S, Vayssières J-F, Kouodiekong L, Woin N. 2008. Inventaire des espèces de mouches des fruits sur goyave dans la région de Yaoundé au Cameroun, Fruits, 63: 19-26.

N'Guetta K. 1994. Inventaire des insectes de fruits récoltés dans le Nord Côte d'Ivoire, Fruits, 49 : 502-503.

Rwomushana R, Ekesi S, Gordon I, Ogol C. 2008. Host Plants and Host Plant Preference Studies for Bactrocera invadens (Diptera: Tephritidae) in Kenya, a New Invasive Fruit Fly Species in Africa. Ann. Entomol. Soc. Am., 101: 331-340.

Tindo M, Tamò M. 1999. Mouche de fruit (Dacus punctatifrons: Diptera, Tephritidae comme problème de production de la tomate dans la région de la Lekié (Sud-Cameroun). Annales Soc. Entomol. France, 35: 525-527.

Van Mele P, Vayssières J-F, Van Tellingen E, Vrolijks J. 2007. Effects of the African weaver ant Oecophylla longinoda in controlling mango fruit flies (Diptera Tephritidae). Journal of Economic Entomology, 100: 695-701.

Vayssières J-F. 2004. Rapport de mission sur l'essai «Piégeage de Tephritidae du manguier» au Sénégal, 11-20 décembre 2004. Rapport CIRAD/ COLEACP-PIP ; 15 p.

Vayssières J-F, Kalabane S. 2000. Inventory and fluctuations of the catches of Diptera Tephritidae associated with mangoes in Coastal Guinea. Fruits, 55: 259-270.

Vayssières J-F, Goergen G, Lokossou O, Dossa P, Akponon C. 2005. A new Bactrocera species in Benin among mango fruit fly (Diptera: Tephritidae) species. Fruits, 60: 371-377.

Vayssières J-F, Korie S, Coulibaly T, Temple L, Boueyi S. 2008. The 
mango tree in northern Benin (1): cultivar inventory, yield assessment, early infested stages and loss due to fruit flies (Diptera Tephritidae). Fruits, 63: 335348.

Vayssières J-F, Korie S, Ayegnon D. 2009a. Correlation of fruit fly (Diptera Tephritidae) infestation of major mango cultivars in Borgou (Benin) with abiotic and biotic factors and assessment of damage. Crop Protection, 28: 477-488.

Vayssières J-F, Sinzogan A, Korie S,

Ouagoussounon I, Thomas-Odjo A. 2009b. Effectiveness of Spinosad Bait Sprays (GF-120) in Controlling Mango-Infesting Fruit Flies (Diptera: Tephritidae) in Benin. Journal of Economical Entomology, 102: 515521.

White IM, Elson-Harris MM. 1992. Fruit Flies of Economic Importance: Their Identification and Bionomics. CAB International: Wallingford, Oxon; $601 \mathrm{p}$. 